\title{
INDUCING INTRINSIC $\gamma$-RAY EMISSION OF THE INTERSTELLAR MEDIUM BY INTENSE FLUXES OF PROTONS AND $\alpha$-PARTICLES IN ACTIVE GALACTIC NUCLEI
}

\author{
D.N. Doikov \\ Odessa National Maritime University. Department of Natural and Technical Sciences \\ doikov@mail.bg
}

\begin{abstract}
Cosmic-ray induced $\gamma$-ray emission in active galactic nuclei (AGN) has been examined in this study for the first time. Cross-sections for the formation of $\gamma$-quanta in such cosmic-ray collisions were selected in the 1-150 MeV energy range. Synthetic $\gamma$-ray spectra were computed for both interstellar gas and dust. At the same energies of particle collisions and induced emission of $\gamma$ quanta, energy intervals of diagnostic interest were determined. Specific characteristics of emission were detected in the energy ranges of 5-15 MeV and 23-30 MeV for most investigated elements. Diffuse continuous $\gamma$-ray spectra for the other energy ranges were less informative with regard to the determination of the chemical composition of interstellar gas. It has been shown that exploring cosmic-ray fluxes in the vicinity of galactic centres by employing the examined $\gamma$-ray spectra yields a pattern of their energy distribution. Diagrams of crosssections for $\gamma$-quanta formation were computed for the $\alpha$ process elements. Synthetic $\gamma$-ray spectra of interstellar gas and dust were calculated individually and collectively. It has been indicated that, under certain conditions in AGN, cross-sections for ionisation of atoms due to energy losses by cosmic-ray induced ionisation and $\gamma$-quanta emission resulting from collisions with cosmic rays are similar. It has been found that when the maximum of proton and $\alpha$ particle energy distribution function falls within the investigated range of energies, it leads to the formation of the peak flux of $\gamma$-rays. This is particularly important for the interpretation of observations in the energy ranges of 5$15 \mathrm{MeV}$ and 23-30 MeV. Synthetic induced $\gamma$-ray spectra of interstellar dust were computed, and methods of their observations for silicate and carbon-containing dust, which account for $80 \%$ and $20 \%$ of the interstellar-dust total mass, respectively, were determined. It has been deduced that the contribution of Compton processes to $\gamma$-quanta emission can be neglected at the investigated energies.
\end{abstract}

Keywords: Active Galactic Nucley, Cosmic Rays, Induced $\gamma$-Ray Spectra, Diffuse Interstellar Medium.

АНОТАЦІЯ. В роботі вперше розглянуто i розраховано індуковане $\gamma$-випромінювання в активних ядрах галактик, яке виникає під дією космічних променів (далі КП). Для інтервалу енергій (1-150 МеВ) підібрані перетини випромінювання $\gamma$-квантів унаслідок зазначених зіткнень. Проведено розрахунки синтетичних $\gamma$-спектрів як для міжзоряного газу, так і для міжзоряного пилу. При цих же енергіях зіткнень частинок та індукування $\gamma$-квантів визначені інтервали енергетичного спектра, що є важливими для діагностики середовища. Визначено, що енергетичні інтервали 5-15 МеВ і 23-30 $\mathrm{MeB}$ дають емісійні особливості для більшості розглянутих елементів. Для інших енергетичних інтервалів маємо менш інформативні для визначення хімічної структури міжзоряного газу дифузні безперервні $\gamma$-спектри. Показано, що в околицях галактичних центрів зондування потоків КП за допомогою розглянутих $\gamma$ спектрів дає структуру їх розподілу по енергіях. Обчислені діаграми перетинів утворення $\gamma$-квантів для елементів $\alpha$-процесу. Синтетичні $\gamma$-спектри міжзоряного газу i пилу визначалися окремо та в сукупності. Зазначено, що за певних умов АЯГ перетину іонізації атомів в результаті іонізаційних втрат, викликаних КП та індукування $\gamma$-квантів під дією зіткнень 3 КП, мають близькі значення. Отримано, що якщо максимум функції розподілу по енергіях у протонів та $\alpha$-частинок припадає на розглянутий нами енергетичний інтервал, то це призводить до утворення максимального потоку енергії $\gamma$-променів. Це особливо важливо для інтерпретації спостережень в інтервалах 5-15 МеВ і 23-30 МеВ. Обчислені синтетичні індуковані $\gamma$-спектри міжзоряного пилу та визначені способи їх спостережень для пилу зі вмістом силікатів та пилу 3 вмістом вуглецю за відповідним співвідношенням як $80 \%$ до $20 \%$ від загальної маси. Отримано, що при розглянутих енергіях внеском $\gamma$-квантів комптоновськими процесами можна знехтувати.

Ключові слова: активне галактичне ядро, космічні промені, індуковані спектри $\gamma$-променів, дифузне міжзоряне середовище.

\section{Introduction}

A distinctive feature of active galactic nuclei (AGN) is their high luminosity in all spectral bands without exception. This is why it is feasible to observe them in many diverse regions of the Universe. On such a scale, we often observe the luminosity produced just by the central region of a distant galaxy, which may extend to a radius of about $1 \mathrm{kpc}$, rather than the integrated luminosity of the entire galaxy. It has been thought up until recently that the total flux of $\gamma$ - and X-rays, being initiated by a galactic central supermassive black hole (GCSMBH), originates only in the region of $1 \mathrm{pc}$, which is quite narrow on a galactic scale. Taking into account the effects of intrinsic 
magnetic fields localised in accretion discs around GCSMBH, it can be deduced that it is only charged cosmic rays with energies not exceeding $3 \mathrm{GeV}$ that, being deviated, return to the galactic centre through the halo (Dermer, 1986). As to the particles of higher energies, they generally leave such galaxies. In our Galaxy, a powerseries semi-empirical energy distribution of protons and $\alpha$ particles is observed along the disc, suggesting that maximum number of particles thus distributed have energies from $5 \mathrm{MeV}$ to $150 \mathrm{MeV}$. The integrated optical and $\mathrm{X}$-ray luminosity at $1 \mathrm{keV}$ and $\gamma$-ray luminosity at 1 $\mathrm{GeV}$ is close to $10^{46}-10^{47} \mathrm{erg} \mathrm{sec}^{-1}$. This has been indicated in the study (Fan et al., 2016), wherein observational catalogue data obtained with different space telescopes are presented and cross-correlated. In particular, they collected and processed luminosities at the aforeindicated energies from catalogues of BL Lacertae objects (BL Lac type of AGN). In this case, having observed luminosities available, it is reasonable to consider the kinetics of collisions of protons and $\alpha$-particles with target atoms of the interstellar medium in AGN, resulting in induced emission of $\gamma$-rays of nuclear-reaction origin. Moreover, in this case, precise boundary conditions of the physical problem of proton and $\alpha$-particle collisions are known. Astrophysical interest in such processes lies in the fact that they are quasi-elastic in the range of collision energies from $5 \mathrm{MeV}$ to $150 \mathrm{MeV}$ with induced $\gamma$-quanta produced in these collisions, among other products. Experimental cross-sections for such reactions have been selected and adopted by the author from the nuclear data services (Tanaka et al. 1994) and reported in the present paper. Even though there are some peculiarities in the induced $\gamma$-ray spectra, it will be deduced from the figures presented below that these spectra are continuous. The peculiarities per se are not narrow nuclear resonances as they cover quite a wide range of energies.

Processes of cosmic rays colliding with atoms of the interstellar medium become explicitly inelastic at energies reaching 1-3 GeV and produce pions, electrons and positrons in proportions $0.25: 0.25: 0.5$. Positrons, in turn, produce only two $\gamma$-ray lines (at $0.511 \mathrm{MeV}$ or $1.022 \mathrm{MeV}$ ) while the neutral pion decay generates a single $\gamma$-ray line at $67.5 \mathrm{MeV}$. Therefore, a distinctive feature of intense fluxes of cosmic rays is that they are associated with continuous, collisioninduced diffuse $\gamma$-ray spectra, as well as $\gamma$-ray lines, commonly attributed to products of series of nuclear decays of $\Delta$-resonance via the following channels (Dermer 1986):

$$
\begin{aligned}
& p+H \rightarrow \pi^{-}+\cdots \rightarrow e^{-}+\cdots(25 \%) ; \\
& p+H \rightarrow \pi^{+}+\cdots \rightarrow e^{+}+\cdots(50 \%) \\
& p+H \rightarrow \pi^{0}+\cdots \rightarrow 2 \gamma(25 \%)
\end{aligned}
$$

The intensities of cosmic-ray fluxes falling on a square centimetre along discs and halo of such galaxies are $10^{8}-$ $10^{10}$ higher than those of similar fluxes in our Galaxy (one particle per square centimetre). Cosmic ray transport from the central to peripheral regions of AGN is described in sufficient detail in a number of papers (Lazarian \& Yan, 2002; Yan \& Lazarian, 2004; Yan, 2015). The takeaway of these studies is the detection of a high degree of scattering cosmic-ray fluxes directed from the AGN centres by large-scale turbulent structures of galactic discs. Thus, on a scale of up to $1 \mathrm{kpc}$, cosmic-ray fluxes have time to become diffuse, whether it is the galactic disc or halo that they are transported through. Consequently, the induced $\gamma$-ray emission described above arises throughout the periphery of AGN.

The present paper consists of five sections, including Introduction, three main sections and Summary. Sections 2 and 3 provide the results of computing synthetic $\gamma$-ray spectra induced by interstellar gas and dust both individually and collectively. Section 4 presents the discussion and conclusions. We introduce new criteria obtained for processing observational data on the composition of cosmic rays and $\gamma$-ray spectra of AGN adopted from available catalogues of the FERMI and PAMELLA missions. Also, the physical nature of the investigated phenomena in AGN is discussed.

\section{2. $\Gamma$-ray emission induced by interstellar atoms in AGN}

As shown in the previous section, the main contribution to the processes of inducing $\gamma$-quanta emission is made by collisions of protons and $\alpha$-particles with atoms in the interstellar medium. The contribution to the similar process by the leptonic component, associated with electrons and positrons, can be neglected. Thus, we obtain the most common reactions, which look as follows:

$$
\begin{aligned}
& p+{ }_{2}^{4} \mathrm{He} \rightarrow \gamma+\cdots \\
& \alpha+{ }_{1}^{1} \mathrm{H} \rightarrow \gamma+\cdots ; \quad \alpha+{ }_{2}^{4} \mathrm{He} \rightarrow \gamma+\cdots
\end{aligned}
$$

Cross-sections for the reactions responsible for producing $\gamma$-quanta have been collected from miscellaneous sources and represented graphically below. The average distribution of matter in AGN from the central to peripheral regions varies within the number density range: $n_{\text {ism }} \approx\left(10^{8}-10^{4}\right) \mathrm{cm}^{-3}$. Fluxes of protons and $\alpha$ particles, $I_{p}\left(R_{\text {per }}\right)$ and $I_{\alpha}\left(R_{\text {per }}\right)$, depending on the distance from the AGN centre, $R_{p e r}$, can be written as the following simplified relationships:

$I_{p}\left(R_{\text {per }}\right)=I_{p 0}\left(R_{\text {per }}\right) / R_{\text {per }}^{2} ; I_{\alpha}\left(R_{\text {per }}\right)=I_{\alpha 0}\left(R_{\text {per }}\right) / R_{\text {per }}^{2}$

The energy spectrum of cosmic rays, $L_{P}\left(R_{\text {per }}\right), L_{a}\left(R_{\text {per }}\right)$, follows a power law. As compared to cosmic rays in our Galaxy, the spectra $L_{P}\left(R_{\text {per }}\right), L_{a}\left(R_{\text {per }}\right)$ are quite similar, having the same power, but different initial energies of particles. This is why, as an initial approximation, the powers for the afore-indicated distributions will be the same while initial distributions will be adopted from observational catalogues in similar fashion as described in the study (Fan et al. 2016). The final stage of determining the intensity of energy flux at a given wavelength is to establish a relationship between cross-sections for the processes of generation of secondary (induced) $\gamma$-quanta and collision energies of protons, $\sigma \gamma(\mathrm{E})$, and $\alpha$-particles, $\sigma \alpha(\mathrm{E})$.

In the figures $1-10$ we represent graphically the dependences of the target cross-sections on the energies $E$ of the relevant collisions with interstellar atoms in AGN. The cross-sections $\sigma \gamma$ and $\sigma \alpha$, represented along the $\mathrm{Y}$-axis, are expressed in millibarns $\left(1 \mathrm{mbn}=10^{-24} \mathrm{~cm}^{2}\right)$. As to the cross-sections for the yield of the target induced (secondary) $\gamma$-quanta, the energy $\mathrm{E}$ is expressed in $\mathrm{MeV}$. The other 
chemical elements in Mendeleev's periodic table exhibit rather low number densities, hence their induced spectra are only considered in some specific cases; in particular, in gasand-dust aggregates. Carbonates and silicates comprise $20 \%$ and $80 \%$, respectively, of interstellar dust grains. Dust grains account for $1 \%$ of the mass of the matter in between interstellar clouds. The fraction of dust inside dense clouds is several times larger due to the dust fragmentation. Therefore, it is reasonable to expect observing induced $\gamma$-ray emission, produced in interactions of dust grains with hadronic component of intense cosmic-ray fluxes. Cosmicray particles contain such elements as $\mathrm{C}, \mathrm{N}, \mathrm{O}, \mathrm{Si}, \mathrm{Al}, \mathrm{Mg}$, $\mathrm{Mn}, \mathrm{S}$ and Fe. Optical, IR and radio spectra have served as the primary tool for studying the emission from dust grains until recently. Some interest in regard to intrinsic spectra of dust has only arisen after the Hubble Space Telescope (HST) mission discovered the soft X-ray scattering by microscopic particles (nanoparticles) produced in Novae (white dwarfs). In the present paper, hard X-ray and $\gamma$-ray emission from dust is considered to be exclusively induced. The $\gamma$-ray background produced by interstellar atoms contrasts with induced emission from dust in gas-and-dust aggregates, since the abundances of the afore-listed heavy metals in such dust grains are relatively higher. Observations of AGN performed by the FERMI and PAMELA space missions have proven the generation of intense fluxes of cosmic rays therein. In the next section, we will present cross-sections and spectra of $\gamma$-ray emission from dust, induced by collisions with such protons and $\alpha$-particles.

\section{Induced $\gamma$-ray emission from interstellar carbon- bearing dust in AGN}

Cosmic-ray dust is comprised of a variety of chemical compounds, with the $\alpha$-process elements predominating over the others. The problem of the formation of its induced $\gamma$-ray emission comes down to the determination of induced $\gamma$-ray emissions from its components. Let us consider systematised cross-sections for induced $\gamma$-quanta from major elements comprising dust grains. With regard to graphite dust, it is sufficient to examine such cross-sections for pure carbon and its most common compounds, including simple oxides and complex compounds, such as carbonates. The mentioned cross-sections are illustrated below with the same legend as in previous figures. A specific spectroscopic feature of the CNO elements is that induced-emission peaks from all these elements are observed at energies 5-15 MeV and 23-30 MeV. In these energy ranges, induced $\gamma$-ray emission reaches its peak while there are distinctive characteristics of the emission typical for particular elements. At energies of 50-150 MeV, the diagnostic potential of the method of induced $\gamma$-ray emission decreases, making it less informative and only suitable for computations and analyses of the mean fluxes of diffuse $\gamma$-ray emission. Therefore, in the energy ranges specified above, the radiation from distant AGN originates not only from their central regions, but also throughout the periphery. It turns out that the presence of intense cosmicray fluxes is a prerequisite for maintaining the AGN luminosity in the $\gamma$-ray energy range.

Studying cosmic rays in our Galaxy has shown that the peak distribution of protons and $\alpha$-particles falls in the investigated range of energies, which results in the induced$\gamma$-quanta yield factor reaching its maximum. In such a case, the procedure of constructing integral induced $\gamma$-ray spectra of AGN is simplified. Prior to strict computation of the induced-emission synthetic spectra, we can estimate the probability of inducing a $\gamma$-quantum, given the boundary conditions of the physical problem.

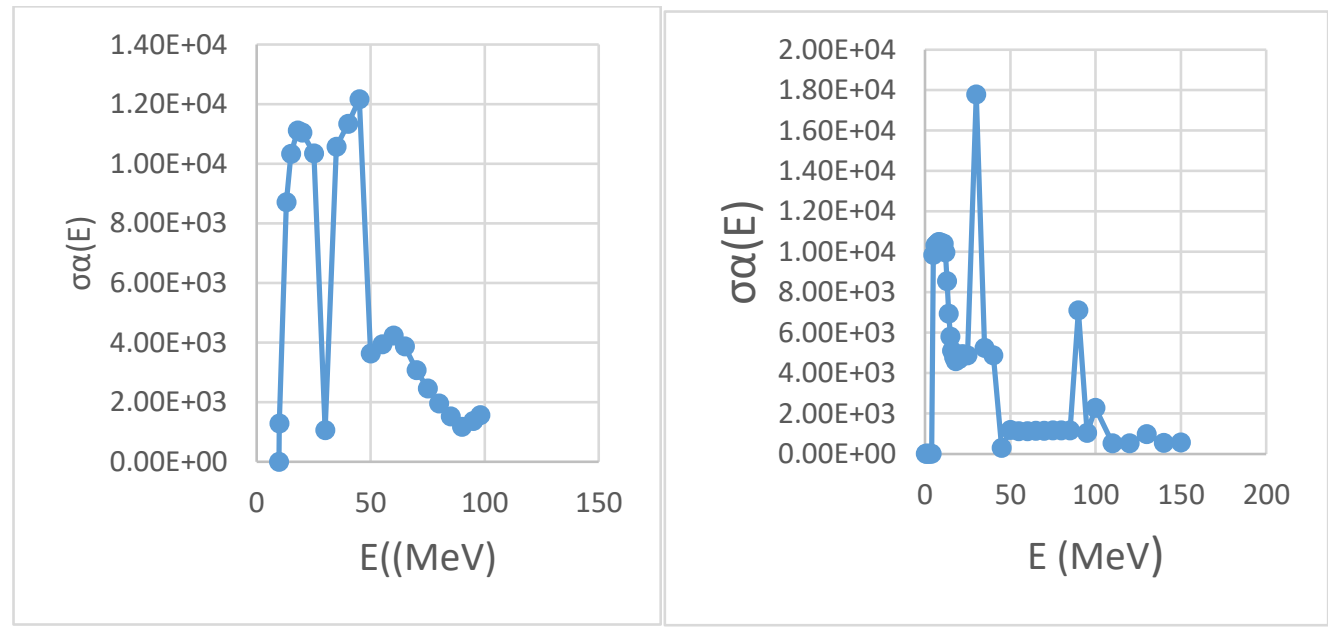

Figure 1: Reaction $\alpha+\mathrm{H}-->\gamma+\ldots$

Figure 2: Reaction $\alpha+\mathrm{He} 4-->\gamma+\ldots$ 


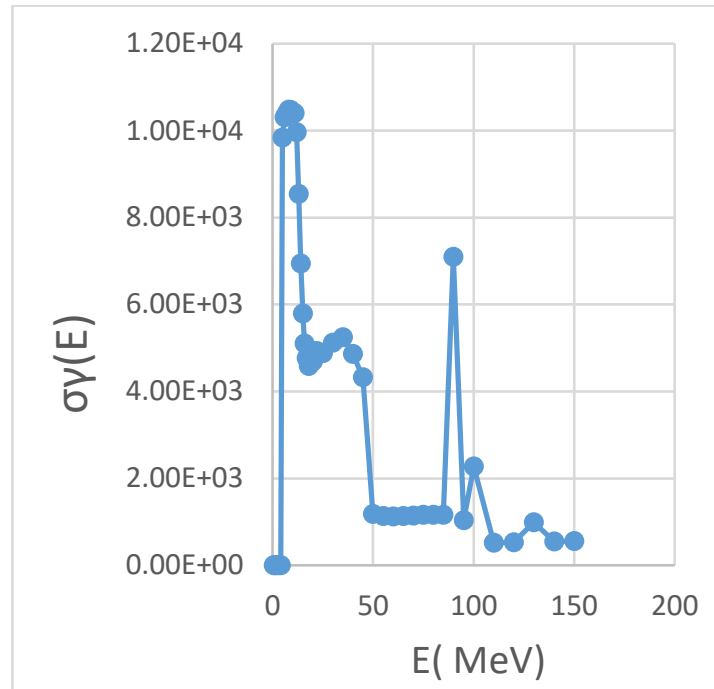

Figure 3: Reaction $\mathrm{p}+\mathrm{C} 12-->\gamma+\ldots$

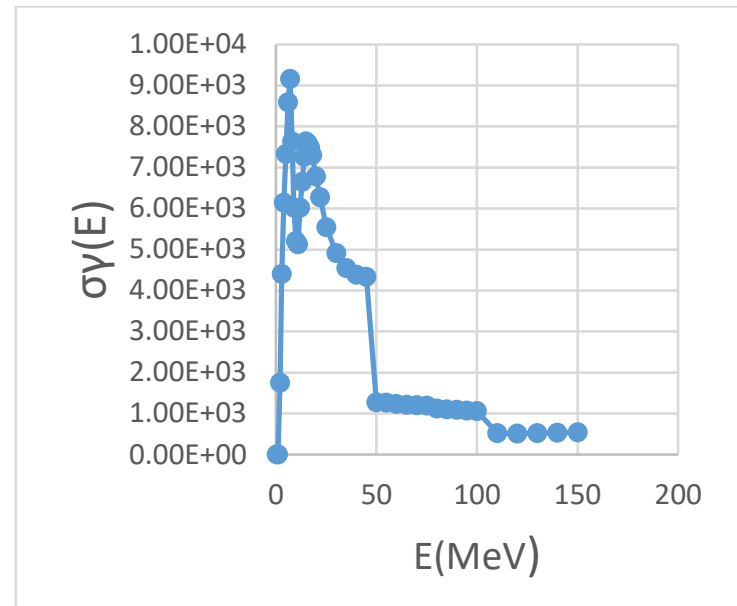

Figure 5: Reaction $\mathrm{p}+\mathrm{C} 13-->\gamma+\ldots$

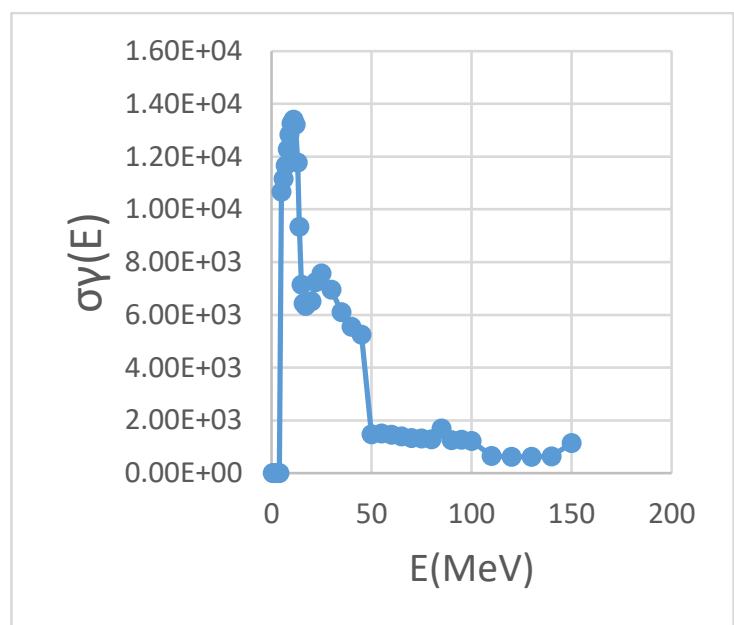

Figure 7: Reaction $\mathrm{p}+\mathrm{O} 16-->\gamma+\ldots$

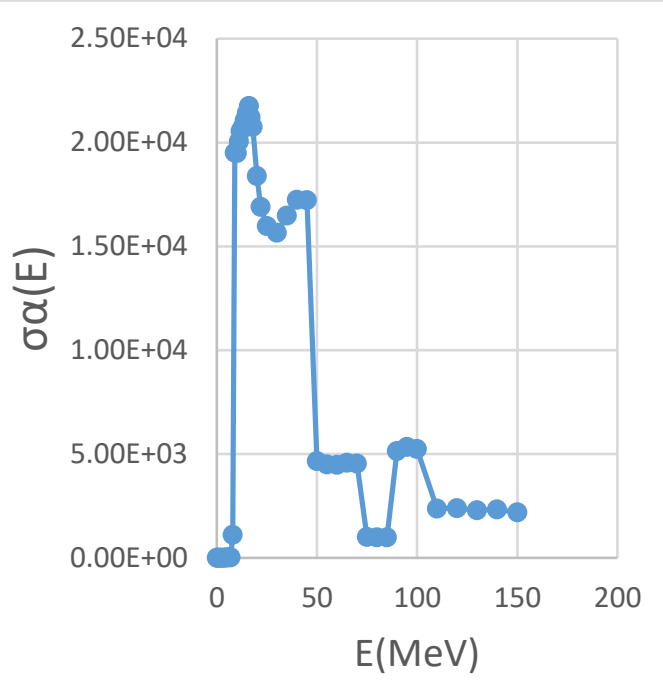

Figure 4: Reaction $\alpha+\mathrm{C} 12-->\gamma+\ldots$

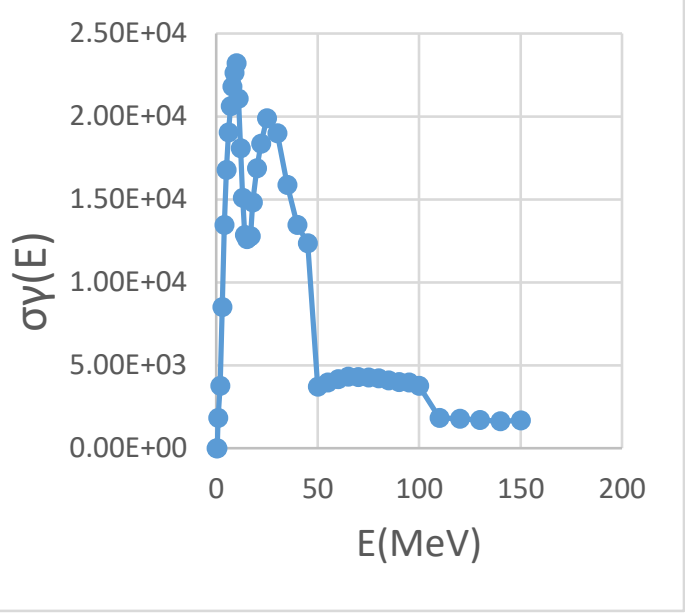

Figure 6: Reaction $\alpha+\mathrm{C} 13-->\gamma+\ldots$

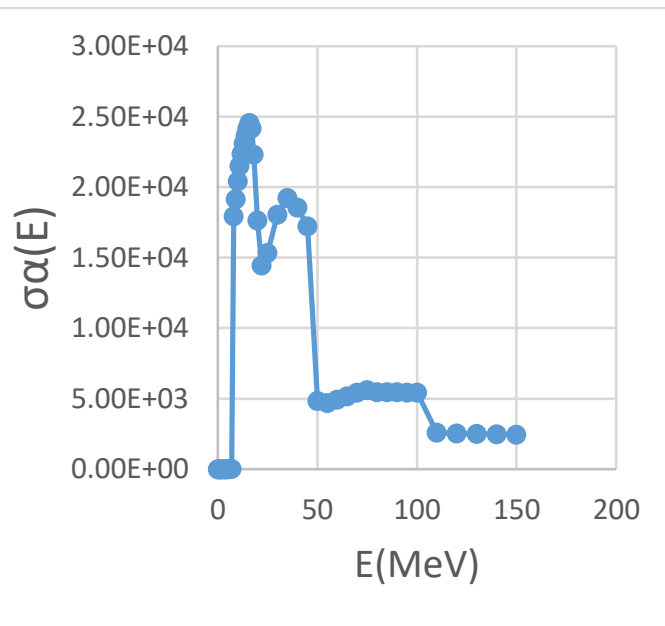

Figure 8: Reaction $\alpha+\mathrm{O} 16-->\gamma+\ldots$ 


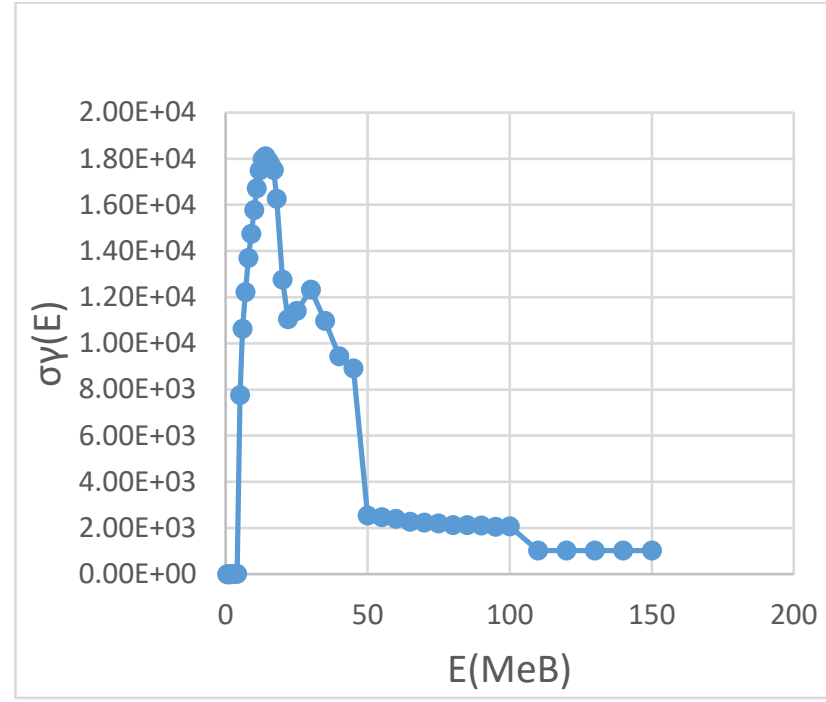

Figure 9: Reaction $\mathrm{p}+\mathrm{Si} 28-->\gamma+\ldots$

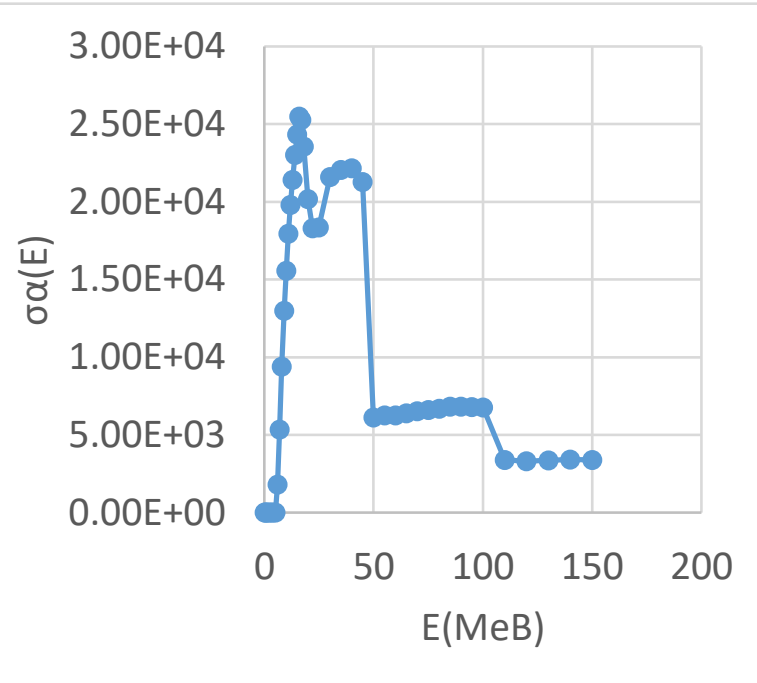

Figure 10: Reaction $\alpha+\operatorname{Si} 28--->\gamma+\ldots$
Physically, such a probability will be equal to 1 when the product $n \sigma_{p} L \geq 1$ or $n \sigma_{\alpha} L \geq 1$. The proposed conditions for interstellar gas are met on the galactic disc scale $(L \approx$ $10^{20} \mathrm{~cm}$ ). As to gas-and-dust aggregates (interstellar clouds), such a condition, given their dimensions on a scale of $L \approx$ $10^{18}-10^{19} \mathrm{~cm}$, is fulfilled for the bulk of interstellar clouds. The concept of cosmic-ray flux in AGN is quite conventional. As mentioned in a series of works (Lazarian \& Yan, 2002; Yan \& Lazarian, 2004; Yan, 2015), high-energy cosmic-ray particles with $E_{C r} \leq 3 \mathrm{GeV}$, being trapped by mean galactic magnetic fields, experience diffuse scattering. The whole bulk of $\gamma$-ray active medium is filled with protons and $\alpha$-particles. Consequently, the appearance of such a medium will be defined by a formula similar to the Osterbrock-Lucy escape probability (OLEP) formula, modified by Városi \& Dwek (Városi \& Dwek, 1999). Computing the radiative transfer in diffuse medium requires preliminary calculation of cross-sections for the absorption (or respective absorption coefficients), $\sigma_{a b}\left(k_{a b}\right)$, and quanta scattering, $\sigma_{s c}\left(\sigma_{s c}\right)$ in the specified energy range. The crosssection $\sigma_{a b}$ was adopted from the study (Kozma \& Fransson, 1992). The cross-section $\sigma_{s c}$ is employed from the previous paper of the author (Doikov 2019).

In the figures presented above, we can see the crosssection for $\gamma$-quanta emission, $\sigma_{e m}$. Then, having computed the value $\omega$, we can define the flux of quanta towards an observer, $F_{E}\left(R_{\text {per }}\right)$.

$\omega=\frac{\sigma_{e m}}{\sigma_{a b}+\sigma_{s c}} ; F_{E}(\tau)=\frac{\pi \epsilon}{\rho \kappa}\left[1-\frac{1}{2 \tau^{2}}+\left(\frac{1}{\tau}+\frac{1}{2 \tau^{2}}\right) e^{-2 \tau}\right]$

The ratio between emissivity and absorptivity per one gram of matter, $\epsilon / \kappa$, corresponds to the ratio of crosssections for emission and absorption (per one atom). Provided that the cosmic-ray flux penetrating the galactic disc is scattered with increasing depth, the emissivity of matter for an external observer will be growing with increasing depth of the disc. Simultaneously, protons and $\alpha-$ particles will be losing their kinetic energies in the normal direction towards the surface proportionally to increasing depth of the disc. In contrast to problems in stellar astrophysics, the number density of matter in galactic discs does not vary significantly in the indicated direction.

It also should be noted that, on a scale of the galactic disc depths, each proton or $\alpha$-particle collides with at least one of the interstellar atoms and generates a single $\gamma$-quantum. When considering a disc as diffusively radiating, we suppose that the optical depth, $\tau$, equals to zero on the surface of such a radiating disk. To simulate graphical representation in future papers, we reduce formula (6) to a form, which is more suitable for computations. In so doing, the cross-section $\sigma_{s c}$ will be reckoned as the cross-section for Compton scattering of $\gamma$-quanta by atomic electrons. In the study (Kozma \& Fransson, 1992), the following ratios have been reported: $\sigma_{a b}(\mathrm{H})=0,06 Z_{H} m_{p} c m^{2}$ for hydrogen and $\sigma_{a b}(\mathrm{He})=$ $0,06 Z_{H e} m_{p} c m^{2}$ for helium. For the rest of elements, the ratio is $\sigma_{a b}(i)=X_{i} 0,06 Z_{i} m_{p} c m^{2}$, where $X_{i}, Z_{i}$ are the abundances and charge numbers of the examined chemical elements. When factoring in the contribution by interstellar dust, we adopt data obtained in the IR studies of the galactic dust, reporting that it is comprised of $20 \%$ of carboncontaining dust and $80 \%$ of silicates.

In conclusion of this chapter we presented the synthetic spectra from all AGN's disc. More specifically, the outflows energetic flux $-F_{\text {out }}(E)$ (Fig. 11) of $\gamma$-quanta created by its periphery, located outside the central zone of 1-2 parsecs. The full synthetic spectra are calculated in $(0,1-150) \mathrm{MeV}$ interval and consists contribution by combination of the induced $\gamma$-ray emission and photoionization, Compton, electron-positron scattering. In this case we used solar abundancies of the AGN's ISM. The probability of $\gamma$-quants escaping from peripheral AGN's galactic disc's a presented in a formula (7) in square brackets, are calculated and presented in Fig. 12. Number densities $-N=10^{4} \mathrm{~cm}^{-3}$ are mean values for AGN's ISM. Galactic discs with $200 \mathrm{pc}$ depths and radial sizes we propose how $\mathrm{R}=400 \mathrm{pc}$. The changing of this AGN's parameters leads to increasing of the energetic flows $F_{\text {out }}(E)$. The Fig. 13 presented optical dependences $\tau(E)$ for this galactic discs. 


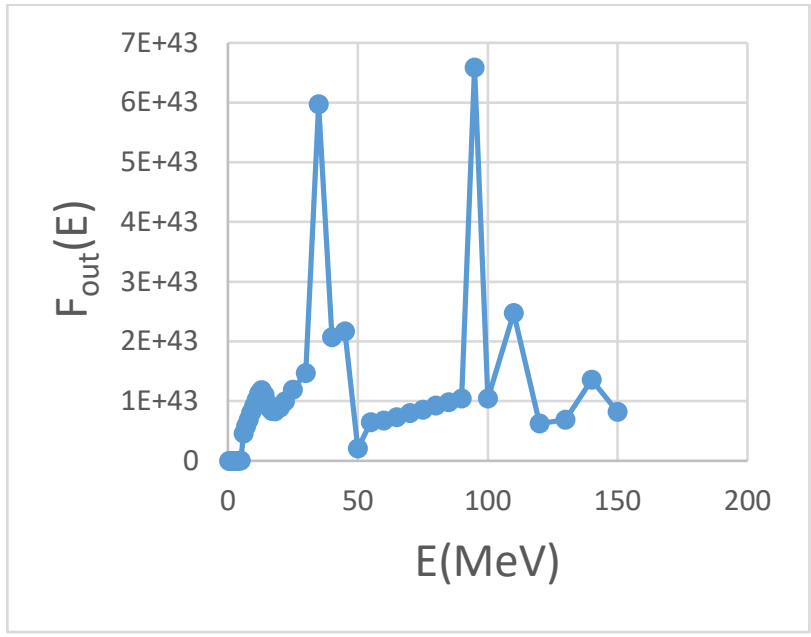

Figure 11: Sinthetic spetra from AGN's ISM with Sun abundancy $F_{\text {out }}(E)$

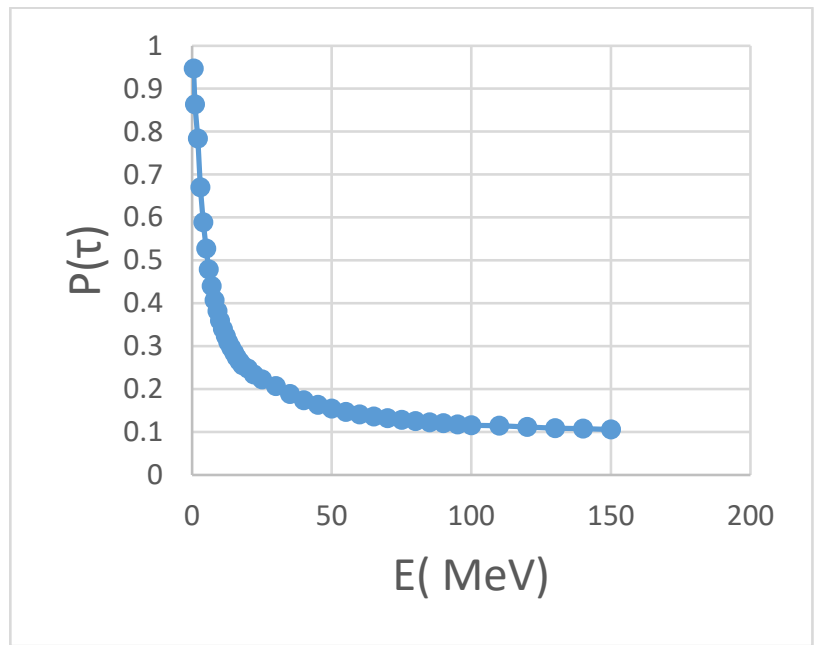

Figure 12: The $\gamma$-ray photon escape probability $\mathrm{P}(\tau)$ from AGN's disc with sun abundances

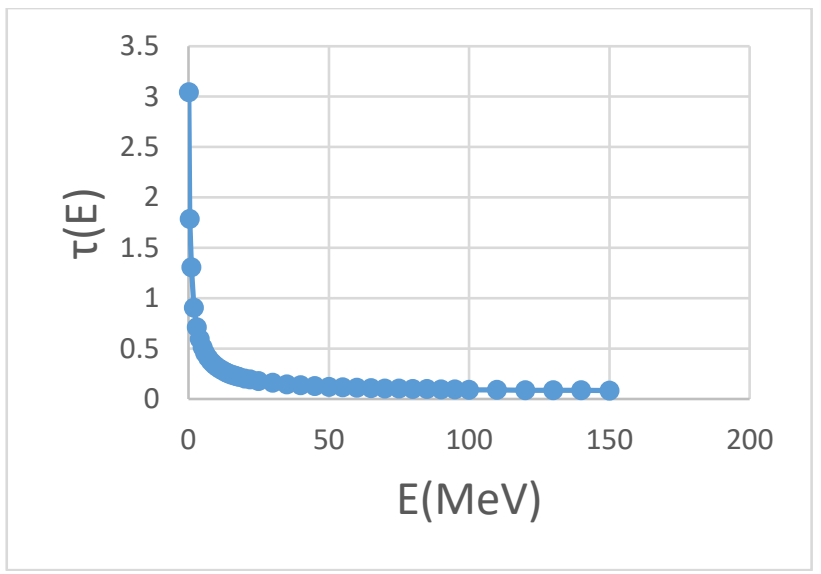

Figure 13: The AGN's ISM optical depth $\tau(\mathrm{E})$ with Sun abundances

\section{Discussion and conclusions}

Measurements of the intensities of hard $\gamma$-ray fluxes from compact sources have been of particular interest recently. In this regard, soft $\gamma$-quanta $0.511-5 \mathrm{MeV}$ provide information mainly about nuclear decays as $\beta$-transformations. Induced emissions in the 5-150 MeV energy range, examined in the present study, result from collisions between cosmic rays and interstellar atoms in AGN. Cross-sections and spectra of such processes, presented in this paper, enabled us to estimate the composition of radiation fields in the peripheral regions of AGN, located at a distance greater than $1 \mathrm{kpc}$, and also to determine its particular spectroscopic value in the region of $5-50 \mathrm{MeV}$, wherein collisions of protons and $\alpha$ particles, comprising cosmic rays, with the $\alpha$-process elements manifest themselves. In other words, spectra of induced $\gamma$-quanta are analogous to continuous stellar spectra. Meanwhile, the nuclear resonance lines, accompanying radiative transitions in such collisions, will be reckoned as analogous to narrow spectral $\gamma$-ray lines. Taking into account the relationship between cosmic-ray fluxes and $\gamma$-ray spectra induced by their collisions, described in the previous sections, the indicated $\gamma$-ray spectra make it possible to reconstruct a dynamic structure of both directed and scattered fluxes of cosmic rays. For distant AGN, the proposed method is the only feasible way to directly study parameters of cosmic-ray fluxes. A portion of energy transferred by the hadronic component of cosmic rays is defined by the structure of accretion discs around supermassive black holes in AGN. Implementation of the Blandford-Znajek mechanism (Blandford \& Znajek, 1977), which describes the acceleration of charged particles around GCSMBH up to the cosmic-ray energies (ranging from $\mathrm{MeV}$ to $\mathrm{GeV}$ ), requires the presence of strong fluctuations in magnetic fields, producing a significant potential difference in the immediate vicinity of a black hole. Integrated observations of AGN in different spectral bands enable us to draw a conclusion about existing correlation between the mass of a black hole, radiative power of the accretion disc and energy output into the surrounding medium. According to observational data, a large majority of AGN can be observed as bright sources of $\gamma$-ray emission. As it follows from the present paper, when there is a correlation between energies associated with such sources of $\gamma$-ray emission and cosmic rays, it results in expected intensities of energy fluxes of $\gamma$-quanta, induced in collisions of cosmic rays with interstellar gas and dust in the 5-150 MeV energy range.

\section{References}

Blandford R.D. and Znajek R.L: 1977, MNRAS, 179, 433. Dermer C.D.: 1986, A\&A, 157, 223.

Doikov D.N., Yushchenko A.V., Jeong Y.: 2019, JASS, Rep. Korea, 36 (1), 21.

Fan J.N., Yang J.H., Liu Y. et al.: 2016, Ap J. Suppl. Ser., 226, A20, 18 pp.

Kozma C., Fransson C.: 1992, Ap.J, 390, 602,

Tanaka S., Yamano N., Hata K. et al.: 1994, Proc. of 8th Int. Conf. on Rad. Shielding, Arlington, April 24-28, 2, 965; Am. Nucl. Soc. Inc.

Városi F., Dwek E.: 1999, Ap. J., 523, 265.

Yan H., Lazarian A.: 2004, Ap. J., 614,757.

Yan H., Lazarian A.: 2002, Ap. J., 566, L105-L108.

Yan H.: 2015, Ap. \& Sp. Sc. Lib., Springer-Verlag Berlin Heidelberg, 407, 253.

Weingarter J.C., Draine B.T., Barr D.K.: 2006, Astrophys. J., 645, 1188 . 